\title{
A Unique Case of Sporadic Creutzfeldt-Jacob Disease Presenting as Progressive Supranuclear Palsy
}

\author{
Munehisa ShImamura, Eiichiro Uyama, Teruyuki Hirano, Tatsuhumi MuraKami, \\ Shuji MitA, Tetsuyuki KITAMOTO* and Makoto UCHINO
}

\begin{abstract}
We report a Japanese case of sporadic CreutzfeldtJakob disease (CJD) presenting as progressive supranuclear palsy. For 2 years after onset, neurological deficits had slowly progressed but neither myoclonus nor periodic synchronous discharge was observed. Diffusionweighted image (DWI) showed unique high signal lesions in the bilateral frontal cortex, left parietooccipital and occipital cortices, but there was nearly no change eight months later. Needle biopsy revealed deposition of prion protein of a patchy/perivacuolar type with spongiform degeneration. Thus, the phenotype of sporadic CJD seems variable and DWI should be performed, even in atypical cases lacking the characteristics of CJD. (Internal Medicine 42: 195-198, 2003)

Key words: Creutzfeldt-Jakob disease, progressive supranuclear palsy, diffusion-weighted image, genetics, pathology, single photon emission computed tomography
\end{abstract}

\section{Introduction}

Sporadic Creutzfeldt-Jakob disease (CJD) is a fatal human transmissible spongiform encephalopathy. The diagnosis of CJD is usually confirmed by the presence of spongiform degeneration with deposition of prion protein $(\mathrm{PrP})$, particularly in the cerebral cortex (1). Approximately, $90 \%$ of cases show common features such as subacute dementia, myoclonus, periodic synchronous discharge (PSD) on electroencephalogram (2), and neuronal protein (14-3-3, $\gamma$ enolase) in cerebrospinal fluid (CSF)(3), and usually die within a year of onset (2). However, there are various pheno- types in the disease and the diagnosis of CJD is sometimes difficult. For example, some cases show symptoms like progressive subnuclear palsy (PSP) in the early stage and survive more than 2 years (4-6). To clarify this issue, recently the effectiveness of diffusion-weighted image (DWI) has been suggested(7-9). Some cases of CJD have been reported to show an abnormally high signal lesion on DWI in the initial stage of CJD(7-9).

Here, we describe a Japanese case of sporadic CJD presenting as supranuclear gaze palsy in the initial stage. In particular, this case showed unique abnormal lesions on DWI without any characteristics of CJD during the disease course of more than 2 years.

\section{Case Report}

A 56-year-old Japanese woman was in good health until two years before evaluation, when her family noticed that she had difficulty in walking at a normal speed. She gradually developed transient difficulties in initiating movement and postural instability walking in small steps. She had unexplained falls, especially backwards. From age 55, her functional deterioration with respect to speaking and handwriting became progressively more evident. She had no history of blood transfusion, or implanting of corneal or dura matter grafts.

On admission, her mini mental-state examination (MMSE) score was 22/30. She showed a mask-like face, supranuclear gaze palsy in the vertical sides, dysarthria, bradykinesia, and retrocollis. There were rigidities in the neck and right upper arm, dysdiadochokinesis, bradykinesia, freezing phenomenon on gait, postural instability, and reduced arm swings. Sensory examinations and deep tendon reflexes were normal. Cerebrospinal fluid analyses including neuron-specific enolase and 14-3-3 protein were normal. EEG showed diffuse $\theta$ wave without PSD. A 1.5 tesla mag-

From the Department of Neurology, Kumamoto University School of Medicine, Kumamoto and *the Department of Neurological Science, Tohoku University School of Medicine, Sendai

Received for publicaton July 17, 2002; Accepted for publication November 25, 2002

Reprint requests should be addrressed to Dr. Munehisa Shimamura, the Division of Gene Therapy Science, Osaka University Medical School, 2-2 Yamadaoka, Suita, Osaka 565-0871 

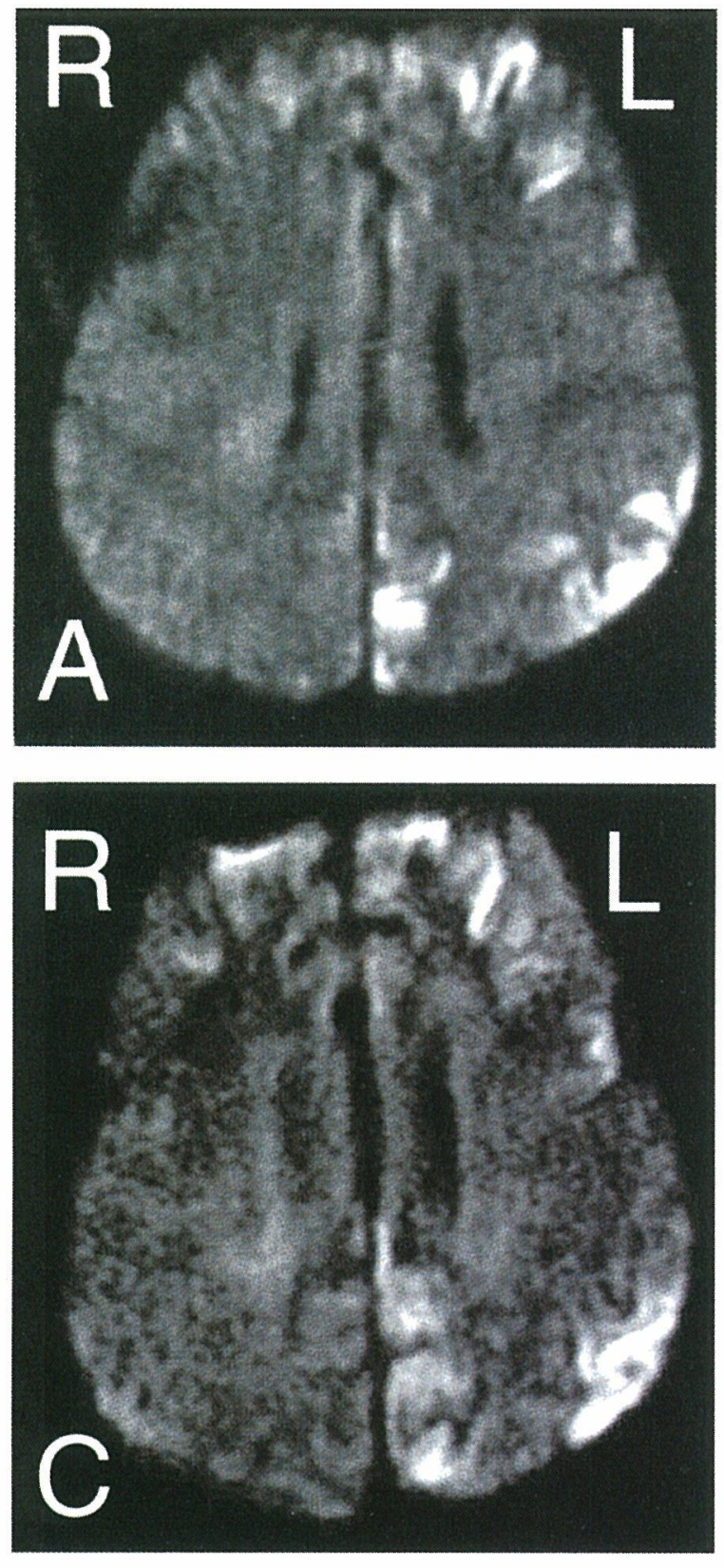

netic resonance imaging (MRI) revealed a mild atrophy in the tegmentum of the midbrain. Single photon emission computed tomography (SPECT) using ${ }^{123}$ I-IMP showed hypoperfusion in the frontal cortex, left parietooccipital cortex and left basal ganglia (Fig. 1B). Although we suspected PSP, we tried treatment with levodopa-carbidopa $200 \mathrm{mg}$ and additional selegiline $10 \mathrm{mg}$ in a day. Her gait disturbance and postural instability improved and she could walk without stumbling. One month later, levodopa-carbidopa and selegiline were withdrawn due to a drug-induced fever. DWI performed two months later revealed high signal lesions in the bilateral frontal cortex, left parietooccipital and occipital cortices (Fig. 1A). Fast fluid attenuated inversion recovery (FLAIR) images showed slightly high signal lesions

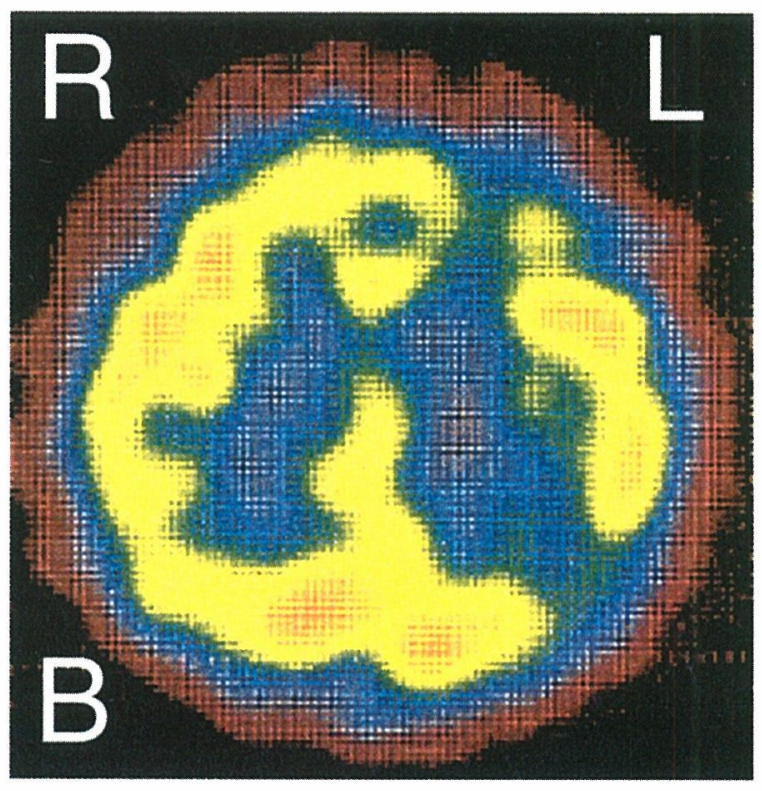

Figure 1. SPECT using ${ }^{123}$ IMP (A) and diffusion-weighted MRI (B, C) at age 56. A: Diffusion-weighted MRI shows high signal lesions in the bilateral frontal cortex and left occipital cortex. B: SPECT image using ${ }^{123}$ I-IMP shows that hypoperfusion corresponded to the abnormalities detected on diffusion-weighted MRI. C: Diffusion-weighted MRI performed eight months later. High signal lesions extended slightly, but were relatively limited to the cerebral cortex.

corresponding to the abnormalities detected on DWI. Apparent diffusion coefficient (ADC) in the cerebral white matter and cerebral angiography were normal. Eight months later, the degree of parkinsonism and supranuclear gaze palsy became more severe and mental deterioration progressed to severe dementia (MMSE 0/30), but neither myoclonus nor PSD were observed. On DWI, abnormal high-signal lesions in the left cerebral cortex had extended a little (Fig. 1C). After informed consent, we performed needle brain biopsy from the left occipital cortex.

Microscopic examination disclosed marked spongiform degeneration consisting of mild gliosis and neuronal loss in areas with no physiolosical change of needle biopsy. In particular, there were massive patchy/perivacuolar PrP-type 

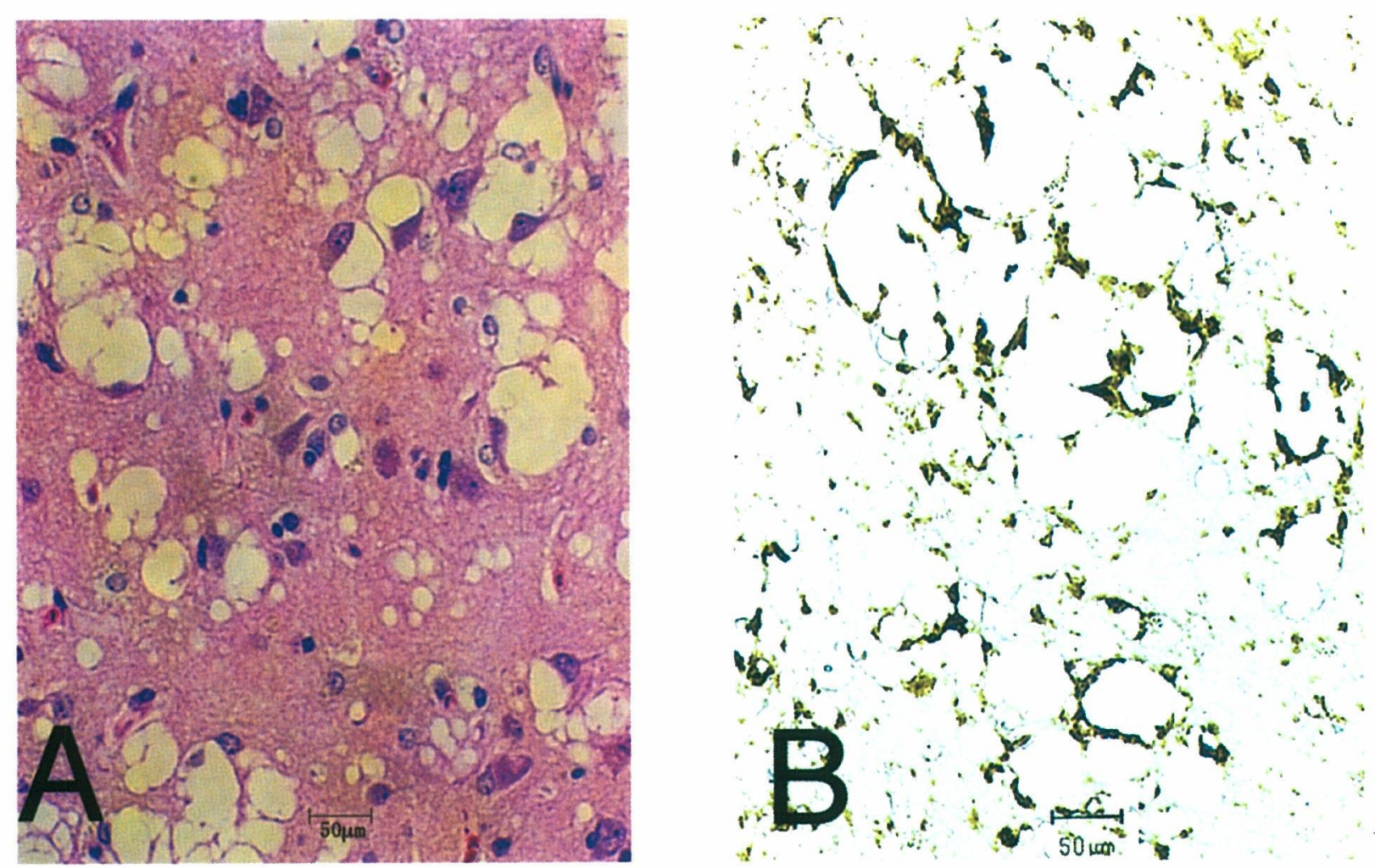

Figure 2. Light micrographs of biopsied tissue from the patient at age 56 (A: HE stain, magnification $\times 400$, B: PrP immunostain, magnification $\times 400)$. A: Marked spongiform change accompanied with neuronal loss and mild gliosis is shown. B: Perivacuolar type-PrP deposits are present.

deposits without florid plaque (Fig. 2). Molecular genetic analysis for prion protein gene (PRNP) revealed no mutation or methionine/methionine homozygosity (MM) at codon 129. We could not perform an immunoblot profile of $\operatorname{PrP}^{s c}$ due to there being too little material from the needle brain biopsy. Although she became bedridden three years from onset, she could still talk and eat by herself.

\section{Discussion}

The diagnosis of sporadic CJD in the present case was established by the presence of spongiform degeneration with patchy/perivacuolar-type PrP deposition in the cerebral cortex. The neuropathological features were identical to sporadic cases of CJD, but the clinical phenotypes and findings on DWI were quite unusual. Recently, signal-intensity changes on DWI were reported to reflect the histologic changes of CJD (7), and show the change in the early stage without any change on conventional MRI (9). The high signal lesion on DWI in the present study was seen in the initial stage without any clinical hallmarks of CJD. Interestingly, the slow extension of the lesion on DWI seems consistent with the slow progression of the clinical course. Compared with other cases, which showed rapid progression of clinical manifestation (7), the extension on DWI is much slower.
Thus, DWI might be effective in the diagnosis and the evaluation of the progression in CJD.

Although disturbance of vertical gaze occurs in 3-5\% cases of CJD (10), a case showing clear L-dopa responsive parkinsonism and supranuclear gaze palsy at the clinical onset is uncommon. Kawasaki et al (5) reported a similar case that showed symptoms like PSP in the early stage and survived more than 4 years. They speculated that their case was the sporadic form of fatal insomnia because the pathology was similar to that of fatal insomnia, which showed a severely affected thalamus and a mutation in codon 178 of the PRNP. It is not clear whether the present case could be classified as sporadic form of fatal insomnia or not, because the thalamus was not investigated pathologically. However, we speculate that the thalamus may not be involved in the present case because there were no clinical manifestations such as sensory impaiment or intractable pain and no lesion on DWI and SPECT. Vingerhoets et al (6) also reported a similar case of sporadic CJD that presented L-dopa responsive parkinsonism and diplopia. In that case, apoptosis in the substantia nigra without PrP deposit was revealed, and they speculated that L-dopa responsive parkinsonism was an early involvement of the nigrostrial dopaminergic pathway. From that viewpoint, the initial clinical signs in the present study could not be explained without the involvement of nigrostrial 
dopaminergic pathway, and we speculate that there were subtle histological changes in that lesion.

A classification of sporadic CJD was proposed on the basis of the genotype at polymorphic codon 129 of PRNP, and the physiochemical properties of the protease-resistant core of the pathological $\operatorname{PrP}^{\mathrm{sc}}(3,11)$. The distinct $\operatorname{PrP}^{\mathrm{sc}}$ isoforms are associated with different patterns of PrP deposition: type 1 is found in areas with diffuse PrP immunoreactivity and type 2 is associated with perivacuolar and plaque-like deposits (12). The present case revealed MM at codon 129 of PRNP that is seen in $96 \%$ of the normal Japanese population. Although it was difficult to identify the type of $\operatorname{PrP}^{\mathrm{SC}}$, we speculate that $\operatorname{PrP}^{\mathrm{SC}}$ in the cortex in the present case might be that of type 2 because of the presence of perivacuolar and plaque-like deposits. However, the classification should be determined by Western blot analysis in other areas, because there are some cases with the cooccurrence of different types of $\mathrm{PrP}^{\mathrm{sc}}$ in the same brain (12).

The present case showed typical signs of PSP at clinical onset. The high signal signs on DWI made us reconsider the diagnosis, which was subsequently proved to be sporadic CJD. Thus, the phenotype of sporadic CJD seems to be more variable and DWI should be performed, even in atypical cases presenting as L-dopa responsive PSP or lacking the characteristics of CJD.

Acknowledgements: The authors are indebted to Katsumi Douura (Faculty of Medicine, Graduate School of Medical Sciences, Kyushu University) for screening 14-3-3 protein.

\section{References}

1) Kretzschmar HA, Ironside JW, DeArmond SJ, Tateishi J. Diagnostic criteria for sporadic Creutzfeldt-Jakob disease. Arch Neurol 53: $913-$ 920, 1996.

2) Brown P, Cathala F, Castaigne P, Gajdusek DC. Creutzfeldt-Jakob disease: clinical analysis of a consecutive series of 230 neuropathologically verified cases. Ann Neurol 20: 597-602, 1986.

3) Parchi P, Giese A, Capellari S, et al. Classification of sporadic Creutzfeldt-Jakob disease based on molecular and phenotypic analysis of 300 subjects. Ann Neurol 46: 224-233, 1999.

4) Will RG, Matthews WB. A retrospective study of Creutzfeldt-Jakob disease in England and Wales 1970-79. I: Clinical features. J Neurol Neurosurg Psychiatry 47: 134-140, 1984.

5) Kawasaki K, Wakabayashi K, Kawakami A, et al. Thalamic form of Creutzfeldt-Jakob disease or fatal insomnia? Report of a sporadic case with normal prion protein genotype. Acta Neuropathol (Berl) 93: 317322, 1997.

6) Vingerhoets FJ, Hegyi I, Aguzzi A, Myers P, Pizzolato G, Landis T. An unusual case of Creutzfeldt-Jakob disease. Neurology 51: 617619, 1998.

7) Demaerel P, Heiner L, Robberecht W, Sciot R, Wilms G. Diffusionweighted MRI in sporadic Creutzfeldt-Jakob disease. Neurology 52: 205-208, 1999.

8) Na DL, Suh CK, Choi SH, et al. Diffusion-weighted magnetic resonance imaging in probable Creutzfeldt-Jakob disease: a clinicalanatomic correlation. Arch Neurol 56: 951-957, 1999.

9) Tribl GG, Strasser G, Zeitlhofer J, et al. Sequential MRI in a case of Creutzfeldt-Jakob disease. Neuroradiology 44: 223-226, 2002.

10) Lueck CJ, Mcllwaine GG, Zeidler M. Creutzfeldt-Jakob disease and the eye. II. Ophthalmic and neuro-ophthalmic features. Eye 14: 291301, 2000.

11) Zerr I, Schulz-Schaeffer WJ, Giese A, et al. Current clinical diagnosis in Creutzfeldt-Jakob disease: identification of uncommon variants. Ann Neurol 48: 323-329, 2000.

12) Puoti G, Giaccone G, Rossi G, Canciani B, Bugiani O, Tagliavini F. Sporadic Creutzfeldt-Jakob disease: co-occurrence of different types of $\operatorname{PrP}(\mathrm{Sc})$ in the same brain. Neurology 53: 2173-2176, 1999. 